\title{
Next 24-Hours Load Forecasting Using Artificial Neural Network (ANN) for the Western Area of Saudi Arabia
}

\author{
A.J. Al-Shareef, E.A. Mohamed ${ }^{1}$ and E.Al-Judaibi ${ }^{2}$ \\ King Abdulaziz University, ${ }^{1}$ \\ Qassim University and ${ }^{2}$ SEC-WOA, Saudi Arabia \\ ealjudaibi@se.com.sa
}

\begin{abstract}
Load forecasting has become in recent years one of the major areas of research in electrical engineering. Most traditional forecasting models and artificial intelligence neural network techniques have been tried out in this task. Artificial neural networks (ANN) have lately received much attention, and a great number of papers have reported successful experiments and practical tests. This paper presents the development of an ANN-based short-term load forecasting model with improved generalization technique for the Regional Power Control Center of Saudi Electricity Company, Western Operation Area (SEC-WOA). The proposed ANN is trained with weather-related data, special events indexes and historical electric load-related data using the data from the calendar years 2003, to 2007 for training. The models tested for one week at two different seasons, typically, summer and Ramadan seasons, the mean absolute average error for day-ahead load forecasting are found $1.57 \%$ and $1.82 \%$ respectively.
\end{abstract}

Keywords: Artificial neural networks, short-term load forecasting, back propagation.

\section{Introduction}

Load forecasting has become in recent years one of the major areas of research in electrical engineering. Load forecasting is however a difficult task. First, because the load series is complex and exhibits several levels of seasonality. Second, the load at a given hour is dependent not only on the load at the previous day, but also on the load at the same hour on the previous day and previous week, and because there are many important exogenous variables that must be considered ${ }^{[1]}$. 
Load forecasting plays an important role in power system planning and operation. Basic operating functions such as unit commitment, economic dispatch, fuel scheduling and unit maintenance, can be performed efficiently with an accurate forecast ${ }^{[2,3-4]}$.

Various statistical forecasting techniques have been applied to short term load forecasting (STLF). Examples of such methods including, Time series $^{[5-6]}$, Similar-day approach ${ }^{[7]}$, Regression methods ${ }^{[5 \& 8]}$ and expert systems ${ }^{[7,9-10]}$. In general, these methods are basically linear models and the load pattern is usually a nonlinear function of the exogenous variables ${ }^{[1]}$. On the other hand, ANN has been proved as a powerful alternative for STLF that it is not relying on human experience. It has been formally demonstrated that ANN's are able to approximate numerically any continuous function to the desired accuracy and it should be expected to model complex nonlinear relationships much better than the traditional linear models that still form the core of the forecaster's methodology. Also, ANN is a data-driven method, in the sense that it is not necessary for the researcher to postulate tentative models and then estimate their parameters. Given a sample of input and output vectors, ANN is able to automatically map the relationship ${ }^{[1,11 \& 12]}$.

This paper presents an ANN model of STLF, typically, next 24hours, for the western area of Saudi Arabia. Time, weather, special season events, and load related inputs are considered in this model. Four years of historical dependent data were used. A special design for the forecasting system that cope with the features of Saudi Arabia (SECWOA) electrical load, is presented.

This paper is organized as follow: Sec II gives an introduction to ANN in general. Sec III shows the load feature of SEC-WOA. The proposed ANN model is described in Sec IV. Test results in Sec V. Conclusions are drawn in Sec VI.

\section{Introduction to ANN}

ANN's are mathematical tools originally inspired by the way the human brain processes information. They are composed of simple elements operating in parallel. These elements are stimulated by biological uneasy systems. As in nature, the network function is determined largely by the connections between elements. You can train an ANN to perform a particular function by adjusting the values of the 
connections (weights) between elements. Commonly ANN's are adjusted, or trained, so that a particular input leads to a specific target output. Therefore, the network is adjusted, based on a comparison of the output and the target, until the network output matches the target (Fig. 1). Typically many such input/target pairs are needed to train a network.

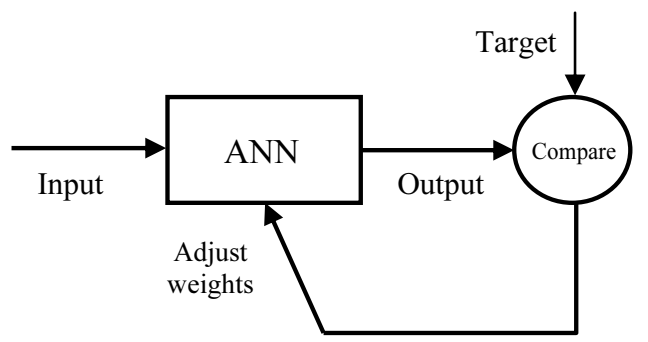

Fig. 1. Simple ANN concept.

Their basic unit is the artificial neuron. The neuron receives (numerical) information through a number of input nodes, processes it internally, and puts out a response. The processing is usually done in two stages: first, the input values are linearly combined, and then the result is used as the argument of a nonlinear activation function. The combination uses the weights attributed to each connection, and a constant bias term. Figure 2 shows one of the most used schemes for a neuron.

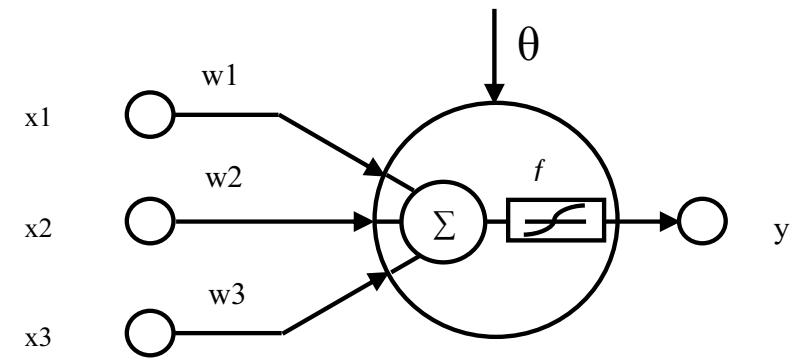

Fig. 2. An artificial neuron.

The neuron output is given by:

$$
y=f\left[\left(\Sigma w_{i}-x_{i}\right)-\theta\right]
$$

Where $x_{i}$ is the neuron input multiplied by weight link $w_{i}, \theta$ is the characteristic neuron offset (bias), and $f$ is the activation function. The most common choice for the activation function in multilayer networks is tan-sigmoid activation function. 
As shown in Fig. 3, the tan-sigmoid activation function output is limited between $[-1,1]$, the output of the function is formulated in (2).

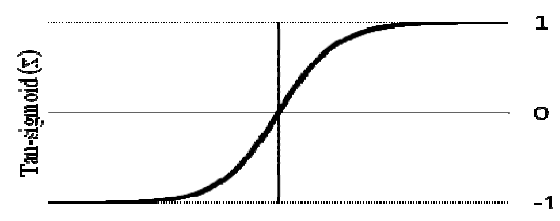

(x)

Fig. 3. Tan-sigmoid activation function.

$$
f(x)=\frac{2}{1+\exp (-2 x)}-1
$$

The neurons are organized in a way that defines the network structure. The most concerned structure is the multilayer perceptron (MLP) type, in which the neurons are organized in layers. The neurons in each layer may share the same inputs, but are not connected to each other. If the architecture is feed-forward, the outputs of one layer are used as the inputs to the following layer. The layers between the input neurons and the output layer are called the hidden layer. Figure 4 shows an example of a two-layers feed-forward perceptron network, with four input neuron, three neurons in the hidden layer and one neuron in the output layer. Each layer has a specified number of nodes; the interconnections are only between neurons of adjacent layers, and each neuron belonging to a layer is connected to all the neurons of adjacent layers. Note that ANN may contain more than one hidden layer; the number of neuron in each layer should be carefully selected depending on the application requirements.

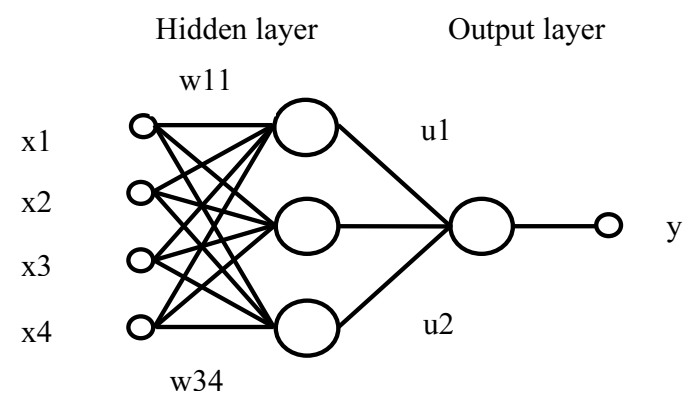

Fig. 4. A two-layer feed-forward neural network. 
The parameters of this network are the weight matrix, and the bias. The estimation of the parameters is called the training of the network. The most used training algorithm in load forecasting is back-propagation one. There are many variations of the back-propagation algorithm. The simplest implementation of back-propagation learning updates the network weights and biases in the direction in which the performance function decreases most rapidly, the negative of the gradient. An iteration of this algorithm can be written in (3).

$$
x_{k+1}=x_{k}-a_{k} g_{k}
$$

Where $x_{k}$ is a vector of current weights and biases, $g_{k}$ is the current gradient, and $\alpha_{k}$ is the learning rate.

In load forecasting applications, this basic form of multilayer feedforward architecture is still the most popular. Nevertheless, there is a large number of other designs, which might be suitable for other applications. ANN should prove to be particularly useful when one has a large amount of data, but little a prior knowledge about the laws that manage the system that generated the data ${ }^{[1,13-14]}$.

\section{Load Feature of SEC-WOA}

Western Operational Area of Saudi Electricity Company is covering very important cities with special features. It includes the two holy mosques in Makkah and Al-Madina, beside, the most economical and touristic city like Jeddah and other small cites such as Taif and Yanbu, Fig. 5 represents the main network of WOA.

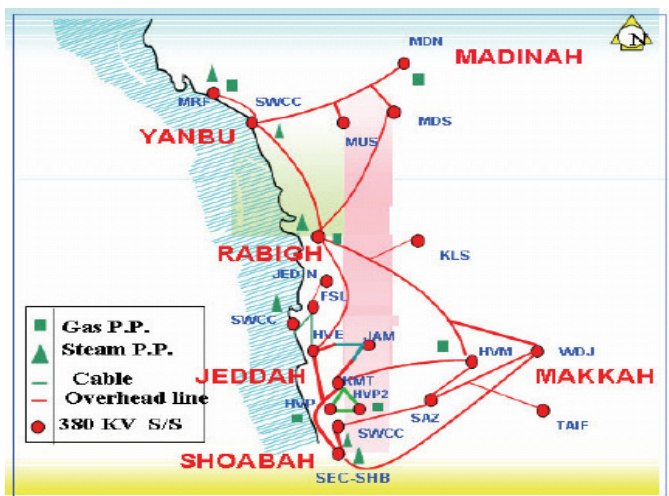

Fig. 5. The main network of WOA. 
There are many factors affecting the load of this area, which make the forecasting unique and challenging.

The weather changes greatly affect the load demand due to a huge air conditioning load in the system. Figure 6 shows a linearity relationship between system daily peak load and related temperature for the year 2004. Another important factor is the time of the day, as social life and activities of the consumers depend on the time of the day such as; working and schools hours and prayer times, In addition to the seasonal load behavior factor, which reflect how load draws a changeable load profile, because of the impact of seasonality. The effect of working days and weekends on the load trend is essential. One more important factor is special events factor mainly religious events such as the month of Ramadan and Hajj, and other events such as public holidays, school and exams. These events, based on the lunar calendar will cause unsimilarity in load conditions every year, so that it has to be considered.

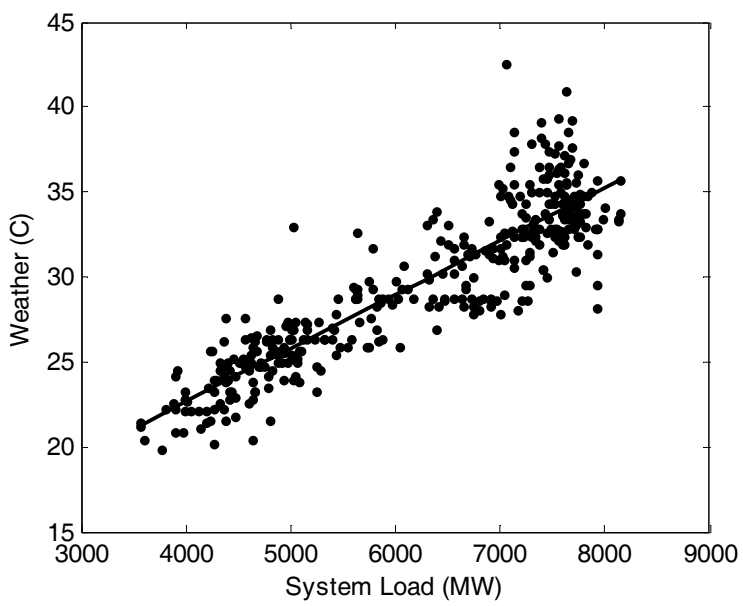

Fig. 6. Relationship between temperature and system daily peak load of 2004.

Examples of a daily load consumption profile of a typical Friday, winter and summer days are shown in Fig. 7. The difference between winter and summer profiles is clear; the effect of hot weather is reflected on the great amount of load consumption at afternoon in the summer day. At Friday a sudden increase in the load demand afternoon is due to Juma 'a prayer, and at weekend the load is stable at morning. 

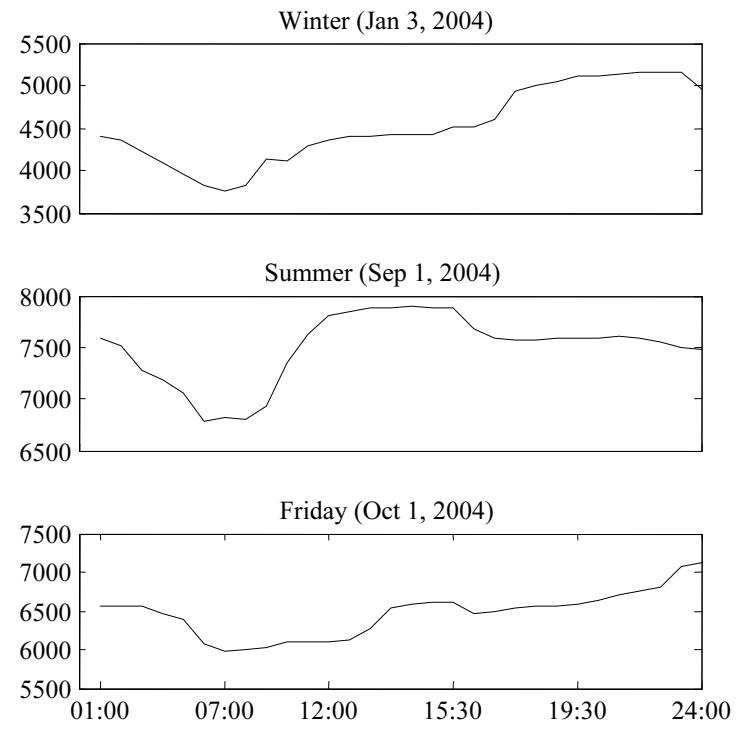

Fig. 7. Examples of daily load consumption profile of a typical Friday, winter and summer days.

\section{The Proposed ANN Model}

The proposed ANN model is a two layers feed-forward ANN, has 22 inputs, one hidden layer with ten neurons, and one neuron in the output layer, with tan-sigmoid activation function in the hidden layer, and linear function in the output layer.

\section{A. Input Vector Configuration}

The input vector to the ANN specifies the date, time, weather, special events data, and associated historical load data for the day to be forecasted, on hourly bases. Typically, it is configured from month, year, hour, seven indexes represent the day type, six indexes represent the special events, temperature, relative humidity and historical load for the same hour of the last three days, and the same day of the last year. Figure 8 depicts the input vector configuration.

Numerical indexes were given to represent the inputs for the forecasted hour. Indexes of [1:12] were given to represent the month, $[1: 24]$ to represent the hour and $[0,1]$ to represent special events and, the day type starting from Saturday to Friday respectively. Moreover, we have considered half-hourly data for the high load variation periods of 
the day, typically $13: 30,14: 30,15: 30,18: 30,19: 30$ and $20: 30$ which were represented by the fractions $(13.5,14.5,15.5,18.5,19.5$ and 20.5) respectively.

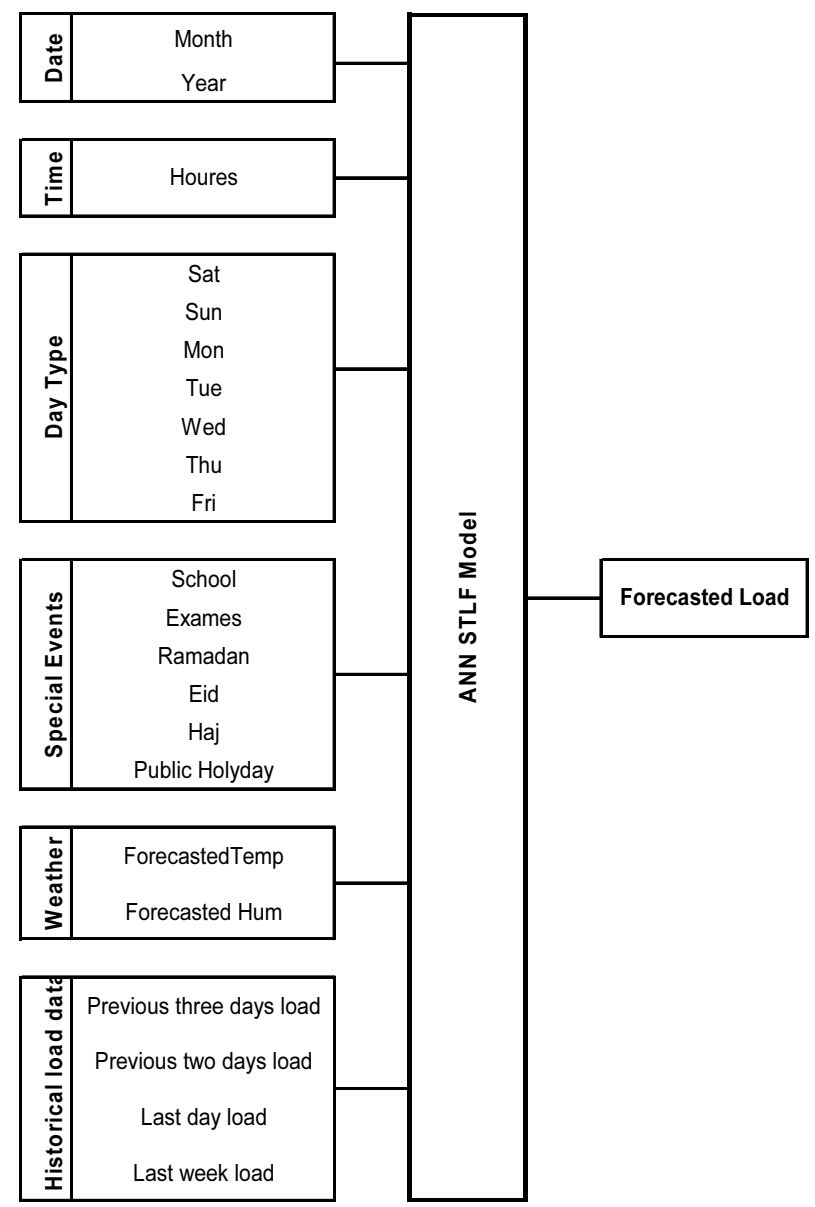

Fig. 8. Input vector configuration.

\section{B. Data Range}

Since the variety of load shows similarity on the same period each year. Therefore, the limits of two months passing the forecasting day, and two months before and after the same day from each of the previous four years were selected as input data range for the training set (Fig. 9) ${ }^{[3,4-15]}$. 


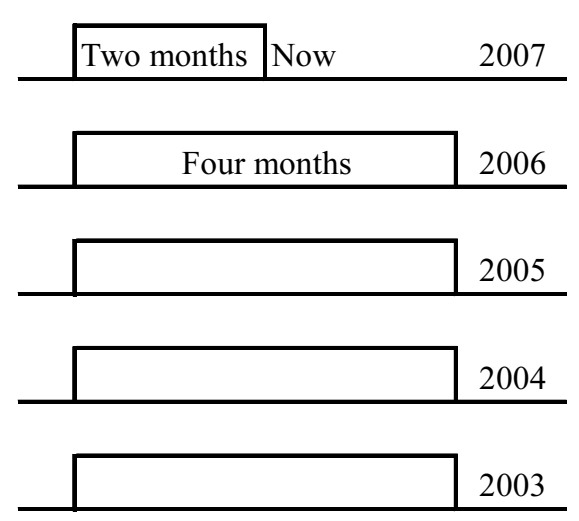

Fig. 9. Limits of input data range for the training set.

\section{Hidden Layer}

In the proposed ANN model, it is found that one hidden layer is enough for the learning of the network. In fact, networks with more than one hidden layer are more complex and time consuming. The number of neurons in the hidden layer has the greatest effect in the network performance. If there are no enough hidden neurons, the network will find difficulty in the learning. On the other hand, the more hidden neurons, the more capability network to over-fit the trained data. In our experiments, varying the number of hidden neurons has led us to the set with the smallest error. Typically, ten neurons in the hidden layer were chosen.

\section{Data Preprocessing}

Before training, it is useful to scale all the inputs and targets so that they always fall within a specified range. Equation (4) is used for each input and targets independently.

$$
y_{s}=\frac{\left(y_{\max }-y_{\min }\right)\left(x-x_{\min }\right)}{x_{\text {max }}-x_{\min }}+y_{\text {min }}
$$

Where $y_{s}$ is the scaled data element, $x$ is the original data element for each input and target vectors, $x_{\max }$ and $x_{\min }$ are the maximum and 
minimum corresponding data element respectively. Due to the nature of the sigmoid function, the outputs of the neurons fall in the interval of $[-1]$ and [+1]. Therefore, $y_{\max }$ and $y_{\min }$ are set to 1 and -1 , respectively ${ }^{[13]}$.

\section{E. Training}

Back propagation algorithm with automated regularization and fixed learning rate is used in this work. The learning rate is multiplied times the negative of the gradient to determine the changes to the weights and biases. If the learning rate is made large, the algorithm becomes unstable. If the learning rate is set small, the algorithm takes a long time to converge. In this work the learning rate is fixed to 0.05 , which shows a good performance.

The main problem in the training is called over-fitting. The error of training becomes very small, but the network will memorize the input data. The network will not be generalized for new test data, and will show a large error when it is tested. To improve the generalization of the network, an automated regularization technique ${ }^{[13]}$ is developed in this model. Typically, the objective of the training process is to reduce the sum of squared error:

$$
\varepsilon_{d}=\sum_{i=1}^{n}\left(t_{i}-\alpha_{i}\right)^{2}
$$

Where $t_{i}$ is the target value, and $\alpha_{i}$ is the network output. However, additional term is added for the regularization, the objective function becomes:

$$
f=\beta \varepsilon_{d}+\alpha \varepsilon_{w}
$$

Where $\varepsilon_{w}$ is the sum of the squared error of the network weights, and $\alpha$ and $\beta$ are the objective function parameters. This performance function causes the network to have smaller weights and biases, which make the network response to be smoother and less likely to overfit. One approach to determine the optimal regularization function parameters in an automated fashion is the Bayesian framework ${ }^{[16,17]}$. 


\section{Test Results}

The performance of the developed model for short-term load forecasting has been tested for one week in two different seasons using the actual load, special events, and weather data (form year 2003 to 2007) for the training. Data was provided by SEC-WOA (Regional Control Center). MATLAB 7.4.0 tools, was used for the numerical simulations.

The load forecast was compared to the actual load data and the error was calculated. The mean absolute percentage error (MAPE) is used to evaluate the performance of these models, it is defined as:

$$
M A P E=\frac{1}{n} \sum_{i=1}^{n} \frac{\left|t_{i}-\alpha_{i}\right|}{t_{i}} \times 100
$$

Where $t i$ is the actual load, ai is the forecasted load, $n$ is the number of data points.

The proposed STLF model was tested for one week starting from Aug 18, 2007. The obtained training performance of the model is shown in Fig. 10, where (a) shows the sum of squared error, (b) shows the sum of squared weights, and the effective number of parameters which is used to update the value of objective function parameters is shown in (c).
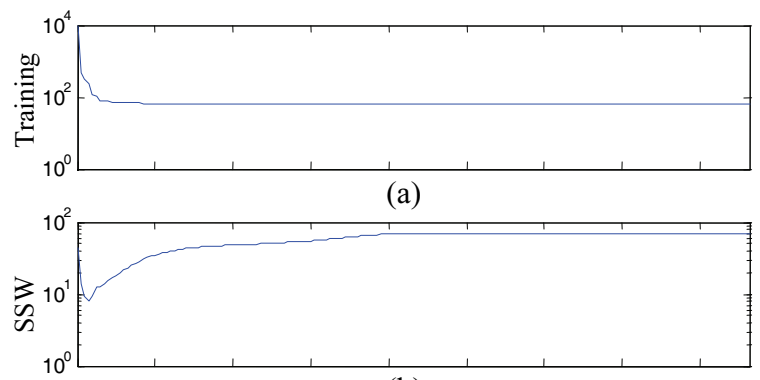

(b)

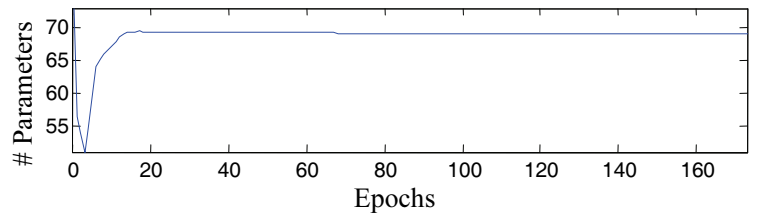

(c)

Fig. 10. Training process; (a) Training SSE , (b) SSW, (c) Effective \# of Parameters. 
The network test results are represented in Fig. 11(I), comparing the network output by the actual load that observes satisfactory forecasting results. MAPE for each hour on each day for this week was obtained $1.57 \%$.

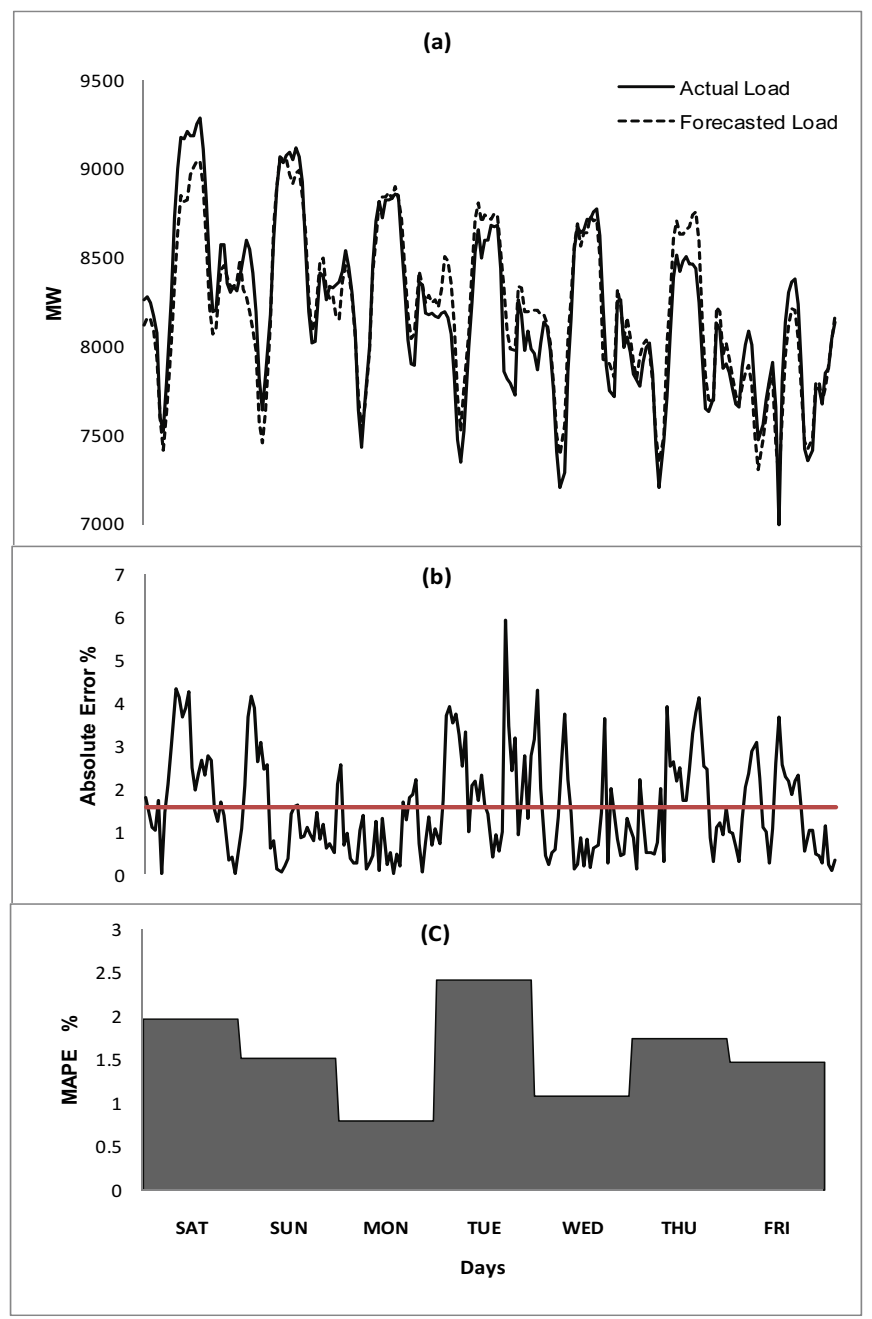

Fig. 11(I). Forecasting results for Aug. 18-24, 2007 (MAPE = 1.57\%); (a) Comparison between hourly actual and forecasted load, (b) hourly absolute error and MAPE, and (c) Daily MAPE.

Also, the model was again tested for another week starting from Sept. 15, 2007 which matches the special event of Ramadan, with a different load profile. The test results are represented in Fig. 11(II) MAPE for each hour on each day for this week was found $1.82 \%$. 


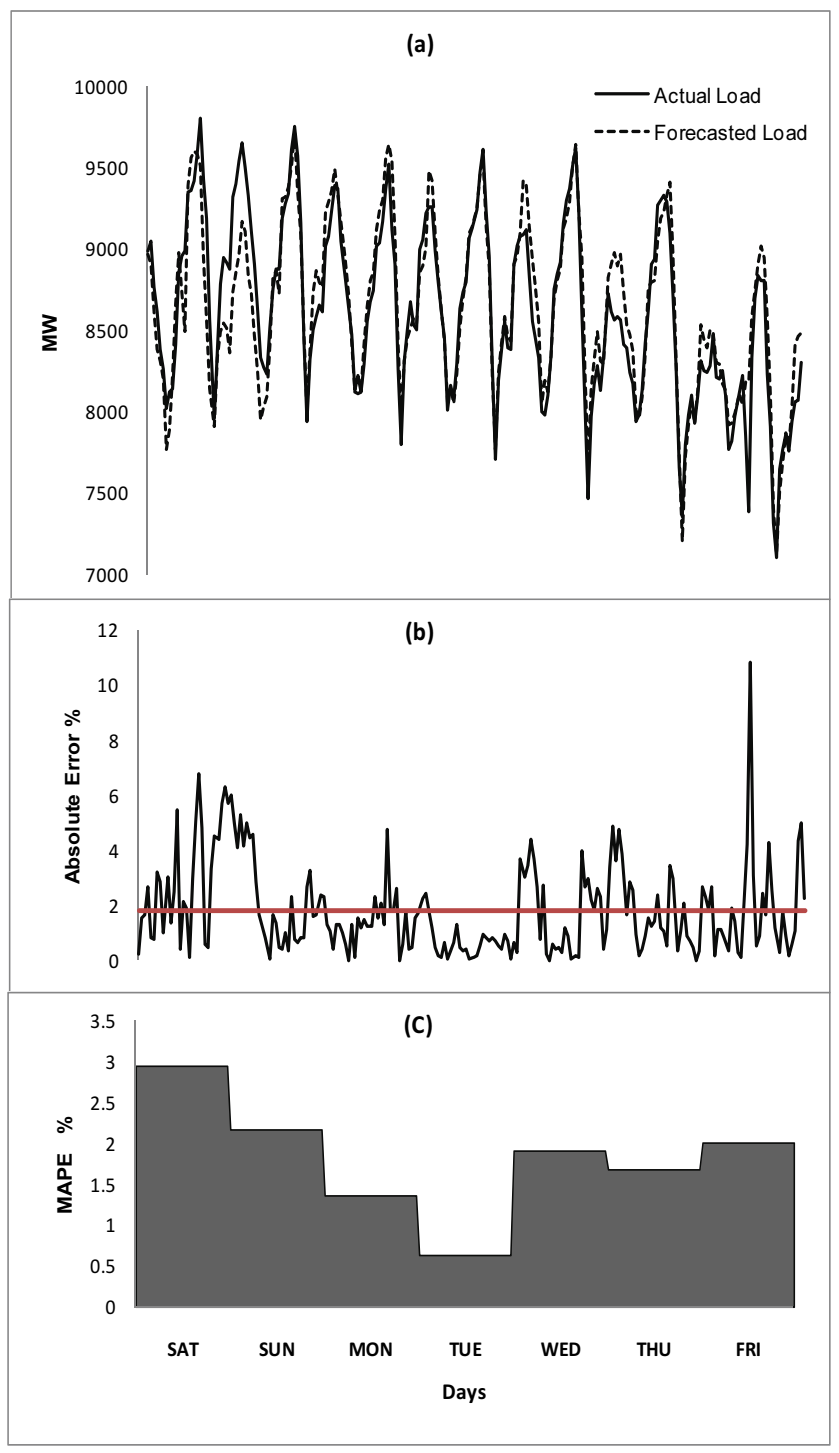

Fig. 11(II). Forecasting results for Sept. 15-21, 2007 (MAPE = 1.82\%); (a) Comparison between hourly actual and forecasted load, (b) hourly absolute error and MAPE, and (c) Daily MAPE.

\section{Conclusion}

This paper presents a new ANN, based STLF application on the load of SEC-WOA network, in the Kingdom of Saudi Arabia. This network 
has a load pattern with special features. These features are to cope with the special religious activities of the Kingdom, such as Ramadan and Hijj. Moreover, the load pattern is much affected by the time schedule of the five prayers a day. Holy cities of Makkah and Madinah host millions of Muslims in different seasons during the year to perform Umra and other religious activities. In fact, the religious tourism influences the system load profile ${ }^{[18]}$. A seasonal ANN structure is also adopted to handle the major contribution of the temperature changes with the four seasons a year, and special events. A simple ANN structure based on the back-propagation and the automated regularization is firstly implemented and secondly evaluated with different scenarios. The mean absolute percentage error (MAPE) for day-ahead load forecasting was $1.57 \%$ and $1.82 \%$ respectively.

Further development of ANN models is to evaluate coupling ANN with particle swarm optimization and wavelet network.

\section{Acknowledgment}

The authors would like to thank SEC-WOA Co., System Operation and Control Department-West, for providing the electrical load and weather data used in this Research.

\section{References}

[1] Hippert, H.S., Pedreira, C.E. and Souza, R.C., "Neural networks for short-term load forecasting: A review and evaluation," IEEE Trans. Power Syst., 16(1): 44-55, Feb. (2001).

[2] Alsayegh, O.A., "Short-term load forecasting using seasonal artificial neural networks," International Journal of Power and Energy Syst., 23(3): 137-142(2003).

[3] Senjyu, T.H., Uezato, T.K. and Funabashi, T., "One hour-ahead load forecasting using neural network," IEEE Trans. Power Syst., 17(1): 113-118, Feb. (2002).

[4] BakIrtzis, A.G., Petrldis, V., Klartzis, S.J., Alexiadls, M.C. and Malssis, A.H., "A neural network short term load forecasting model for the Greek power system," IEEE Trans. Power Syst., 11(2): 858-863, May (1996).

[5] Moghram, I. and Rahman, S., "Analysis and evaluation of five short term load forecasting techniques", IEEE Trans. Power Syst., 4(4): 1484-1491, Nov. (1989).

[6] Lru, K., Subbarayan, S., Shoults, R.R., Manry, M.T., Kwan, C., Lewis, F.L. and Naccarino, J., "Comparison of very short term load forecasting techniques," IEEE Trans. Power Syst., 11(2): 877-882, May (1996).

[7] Drezga, I. and Rahman, S., "Short-term load forecasting with local ANN predictors," IEEE Trans. Power Syst., 14(3): 844-850, Aug. (1999).

[8] Alex, D. and Timothy, C., "A regression-based approach to short term system load forecasting," IEEE Trans. Power Syst., 5(4): 1535-1550, Nov. (1990).

[9] Rahman, S. and Hazim, O., "A generalized knowledge-based short term load forecasting technique”, IEEE Trans. Power Syst., 8(2): 508-514, May (1993). 
[10] Ho, K.L., Hsu, Y.Y., Liang, C.C. and Lai, T.S., "Short-term load forecasting of Taiwan power system using a knowledge-based expert systems", IEEE Trans. Power Syst., 5(4): 1214-1221, Nov. (1990).

[11] Chen, H., Canizares, C.A. and Singh, A. "ANN-based short-term load forecasting in electricity markets", Proceedings of the IEEE Power Engineering Society Transmission and Distribution Conference, 2: 411- 415(2001).

[12] Park, D.C., Elsharqawi, M.A. and Marks, R.J., "Electric load forecasting using artificial neural network", IEEE Trans. Power Syst., 2(3)334-345, May (1991).

[13] Hagan, M.T., Demuth, H.B. and Beale, M.H., "Neural Network Design", Boston, MA: PWS Publishing (1996).

[14] Haykin, S., "Neural Network - a Comprehensive Foundation", 2 Ed., Prentice Hall International.

[15] Senjyu, T., Mandal, P., Uezato, K. and Funabashi, T., "Next day load curve forecasting using hybrid correction method", IEEE Trans. Power Syst., 20(1), Feb. (2005).

[16] MacKay, D.J.C., "Bayesian interpolation", Neural Computation, 4: 415-447(1992).

[17] Foresee, D. and Hagan, F., "Gauss-Newton approximation to Bayesian learning," International Conference on Neural Networks, vol. 3, pp: 1930-1935(1997).

[18] Al-Shareef, A. J., Mohamed, E.A. and Al-Judaibi, E., "One Hour Ahead Load Forecasting Using Artificial Neural Network for the Western Area of Saudi Arabia", International Journal of Electrical Systems Science and Engineering, 1(1). 
التتبو ء بالأحمال على مدى الأربع و العشرين ساعة التالية باستخدام الثبكة العصبية للمنطقة الغربية بالمملكة العربية السعودية

\section{عبد العزيز جلال الثريف، و إ. ا. محمد، و إ. الجديبي}

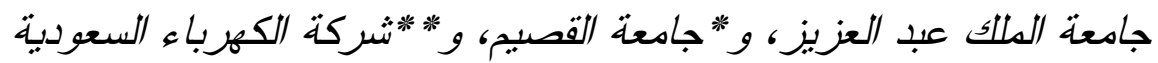
بالمنطقة الغربية، المدلكة العربية السعودية

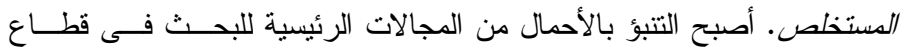

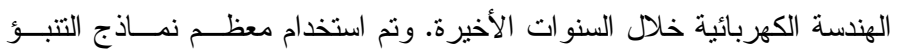

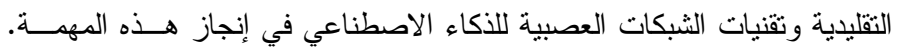

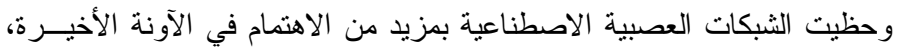

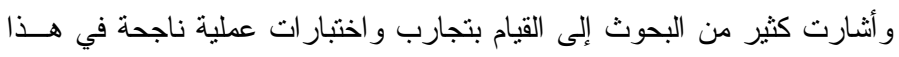

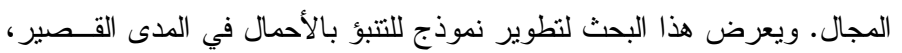

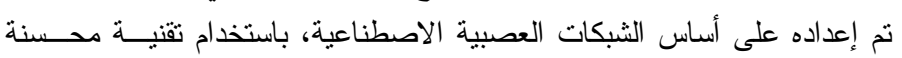

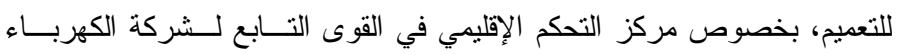

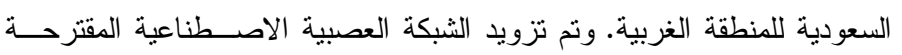

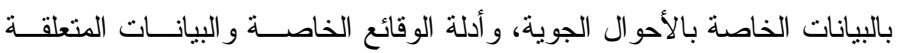

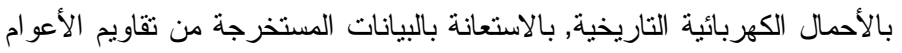

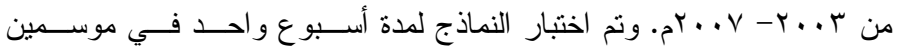

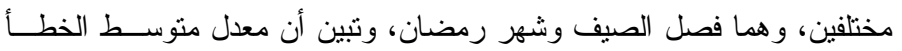

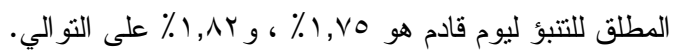

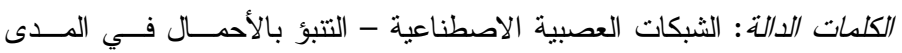

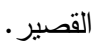

